\title{
Case Report: Thelazia callipaeda Eye Infection: The First Human Case in Germany
}

\author{
Sebastian Dolff, ${ }^{1 *}$ Jan Kehrmann, ${ }^{2}$ Philip Eisermann, ${ }^{3}$ Sami Dalbah,,${ }^{4}$ Dennis Tappe, ${ }^{3}$ and Philipp Rating ${ }^{4}$ \\ ${ }^{1}$ Department of Infectious Diseases, University Hospital Essen, University Duisburg-Essen, Essen, Germany; ${ }^{2}$ Institute of Medical Microbiology, \\ University Hospital Essen, University Duisburg-Essen, Essen, Germany; ${ }^{3}$ National Reference Center for Tropical Pathogens, Bernhard \\ Nocht Institute for Tropical Medicine, Hamburg, Germany; ${ }^{4}$ Department of Ophthalmology, University Hospital Essen, University \\ Duisburg-Essen, Essen, Germany
}

\begin{abstract}
Thelazia callipaeda eye worm is a nematode transmitted by drosophilid flies not only primarily to carnivores and lagomorphs but also to humans. Only a few cases have been reported in Europe (Italy, France, and Portugal). Here, we report the first eye infection in a German patient.
\end{abstract}

\section{CASE REPORT}

Thelazia callipaeda is a vector-borne zoonotic nematode that parasitizes the orbital cavities of domestic and wild carnivores such as foxes, dogs, and cats. ${ }^{1}$ However, rabbits and humans are also infected. Infective larvae of $T$. callipaeda are transmitted by Drosophila flies that feed on lacrimal secretions of infected animals and humans that contain Thelazia spp. firststage larvae. ${ }^{2}$ After transmission, the larvae develop to adult stages in the ocular sac of the vertebrate host within 1 month. ${ }^{2}$ Here, we molecularly identified $T$. callipaeda from an eye infection of a German patient with an extensive travel history.

A 25-year-old woman who lives and works in Germany presented with a foreign body sensation, lacrimation, and redness of her left eye to her local ophthalmologist. The symptoms had lasted for 2 months before she discovered a small transparent filamentous structure in her left eye. She thereupon attended the University Hospital of Essen in November 2018, where the examination revealed subconjunctival congestion of her left eye. A slowly moving worm in the inferior conjunctival fornix was detected by slit-lamp examination. Four worms were extracted immediately. Another worm was removed the next day by forceps under local anesthesia (Figure1A). The anterior segment of her right eye was completely normal. In both eyes, there were no pathologic retinal findings by funduscopy, the intraocular pressure was normal, and visual acuity was $20 / 20$ without correction. Laboratory analyses were without any pathological finding, including complete blood count, urine analysis, and liver and renal function tests. There was no peripheral blood eosinophilia. One ethanol-fixed helminth specimen was subjected to DNA isolation and subsequent nematode 12S rDNA polymerase chain reaction (PCR). ${ }^{3}$ Sanger sequencing of the PCR product revealed $100 \%$ homology to $T$. callipaeda after BLAST analysis (https://blast.ncbi.nlm.nih.gov). The 12S rDNA sequence did not show any heterozygous bases, so it might likely be haploid in 1n; cytochrome oxidase 1 PCR has not been performed. Morphological identification of the worm is illustrated in Figure 1B. During a follow-up visit 2 days later, there were no more worms detected by slit-lamp examination, although the patient reported a nasal tingle on her left eye. On two further follow-up visits (after 1 and 2 weeks), the foreign

*Address correspondence to Sebastian Dolff, Department of Infectious Diseases, University Hospital Essen, Hufelandstr 55, Essen 45122, Germany. E-mail: sebastian.dolff@uk-essen.de body sensation was only occasionally present but the follicles in the left eye were unchanged (Figure 1C).

\section{DISCUSSION}

The patient has been living in an urban area of Germany without contact to pets. She had a travel history including visits to India in 2016, to North America in 2017, and to the Netherlands, Belgium, Luxembourg, Northern Italy, Croatia, Bosnia, Montenegro, Albania, Bulgaria, Romania, Hungary, Slovakia, Austria, and Greece in May-September 2018. On specific questioning, the patient reported ocular fly contact that had occurred during cycling tours while traveling abroad in Eastern Europe in 2018. Several flies had been detected in the conjunctival sac after these cycling tours. The transmission of Thelazia callipaedes is most likely due to lacrimal feeding of the flies in this context. There was no history of local injury or insect bites.

Imported animal cases of thelaziasis have been reported in Germany, the Netherlands, and Switzerland. ${ }^{4,5}$ An autochthonous case in a dog has been reported in Germany ${ }^{6}$ close to the German-French border. The infection is more common in dogs and cats in Italy. ${ }^{7}$ Despite increasing reports of $T$. callipaeda infection in carnivores in different European countries, ocular infections in humans remain rare. ${ }^{8,9}$ However, the number of reported human cases has increased in many countries of Asia, where thelaziasis occurs predominantly in rural areas with poor living conditions and low socioeconomic standards. ${ }^{4}$ Mainly the elderly and children are infected. Remarkably, in the United States, Thelazia californiensis is the only Thelazia species described so far in humans. The vector of this species are little houseflies. Besides, Phortica variegata has been proven to be a competent vector and intermediate host. ${ }^{10}$ In humans, mechanical removal is the treatment of choice, whereas for the treatment of canine infections with $T$. callipaeda, topical installation of organophosphates, and treatment with $1 \%$ moxidectin or $10 \%$ imidacloprid and $2.5 \%$ moxidectin have been effective. In our case, mechanical removal was curative.

Thelaziasis is unknown to most physicians and ophthalmologists, and with this report we seek to raise awareness in the medical community for this infection. Besides dirofilariasis, loiasis, and Onchocerca lupi infection, thelaziasis should be included in the differential diagnosis of ocular helminth disease, particularly in patients with a travel history. ${ }^{11-13}$ 

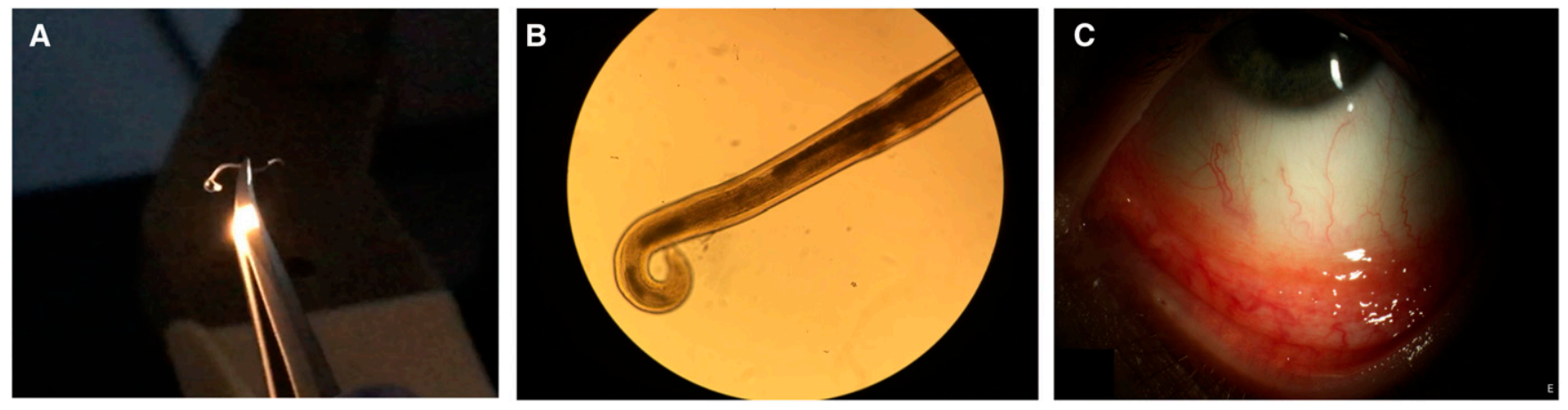

FIGURE 1. Adult stage of Thelazia callipaeda after extraction from the patient's left eye with forceps (A). Morphologic characterization of $T$. callipaeda (B). Slit-lamp photography of the inferior conjunctival fornix (left eye) with chemosis and follicles 2 weeks after worm extraction (C).

Received June 27, 2019. Accepted for publication October 29, 2019. Published online January 6, 2020.

Authors' addresses: Sebastian Dolff, Department of Infectious Diseases, University Hospital Essen, University Duisburg-Essen, Essen, Germany, E-mail: sebastian.dolff@uk-essen.de. Jan Kehrmann, Institute of Medical Microbiology, University Hospital Essen, University Duisburg-Essen, Essen, Germany, E-mail: jan.kehrmann@uk-essen.de. Philip Eisermann and Dennis Tappe, National Reference Center for Tropical Pathogens, Bernhard Nocht Institute for Tropical Medicine, Hamburg, Germany, E-mails: eisermann@bnitm.de and tappe@bnitm.de. Sami Dalbah and Philipp Rating, Department of Ophthalmology, University Hospital Essen, University Duisburg-Essen, Essen, Germany, E-mails: sami. dalbah@uk-essen.de and philipp.rating@uk-essen.de.

\section{REFERENCES}

1. Otranto D, Dantas-Torres F, Mallia E, DiGeronimo PM, Brianti E, Testini G, Traversa D, Lia RP, 2009. Thelazia callipaeda (Spirurida, Thelaziidae) in wild animals: report of new host species and ecological implications. Vet Parasitol 166: 262-267.

2. Otranto D, Lia RP, Buono V, Traversa D, Giangaspero A, 2004. Biology of Thelazia callipaeda (Spirurida, Thelaziidae) eyeworms in naturally infected definitive hosts. Parasitology 129: 627-633.

3. Otranto D, Testini G, De Luca F, Hu M, Shamsi S, Gasser RB, 2005. Analysis of genetic variability within Thelazia callipaeda (Nematoda: Thelazioidea) from Europe and Asia by sequencing and mutation scanning of the mitochondrial cytochrome c oxidase subunit 1 gene. Mol Cell Probes 19: 306-313.

4. Otranto D, Dutto M, 2008. Human thelaziasis, Europe. Emerg Infect Dis 14: 647-649.
5. Malacrida F et al., 2008. Emergence of canine ocular thelaziosis caused by Thelazia callipaeda in southern Switzerland. Vet Parasitol 157: 321-327.

6. Magnis J, Naucke TJ, Mathis A, Deplazes P, Schnyder M, 2010. Local transmission of the eye worm Thelazia callipaeda in southern Germany. Parasitol Res 106: 715-717.

7. Seixas F, Travassos P, Coutinho T, Lopes AP, Latrofa MS, Pires MDA, Cardoso L, Otranto D, 2018. The eyeworm Thelazia callipaeda in Portugal: current status of infection in pets and wild mammals and case report in a beech marten (Martes foina). Vet Parasitol 252: 163-166.

8. Mihalca AD, D'Amico G, Scurtu I, Chirila R, Matei IA, Ionica AM, 2015. Further spreading of canine oriental eyeworm in Europe: first report of Thelazia callipaeda in Romania. Parasit Vectors 8: 48.

9. Colella V, Kirkova Z, Fok E, Mihalca AD, Tasic-Otasevic S, Hodzic A, Dantas-Torres F, Otranto D, 2016. Increase in eyeworm infections in eastern Europe. Emerg Infect Dis 22: 1513-1515.

10. Otranto D, latta R, Lia RP, Cavalera MA, Maca J, Pombi M, DantasTorres F, Jaenike J, 2018. Competence of Phortica variegata from the United States as an intermediate host of the Thelazia callipaeda eyeworm. Am J Trop Med Hyg 98: 1175-1178.

11. Tappe D, Plauth M, Bauer T, Muntau B, Diessel L, Tannich E, Herrmann-Trost P, 2014. A case of autochthonous human Dirofilaria infection, Germany, March 2014. Euro Surveill 19: 2-4.

12. Antinori $S$ et al., 2012. Imported Loa loa filariasis: three cases and a review of cases reported in non-endemic countries in the past 25 years. Int $J$ Infect Dis 16: e649-e662.

13. Bergua A, Hohberger B, Held J, Muntau B, Tannich E, Tappe D, 2015. Human case of Onchocerca lupi infection, Germany, August 2014. Euro Surveill 20: 21099. 